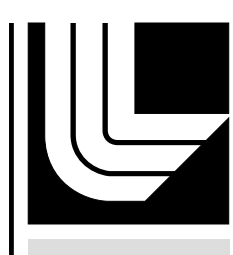

LAWRENCE LIVERMORE N A T IO N A L LABORATORY

\title{
Low Mode Control of Cryogenic ICF Fuel Layers Using Infrared Heating
}

R. A. London, B. J. Kozioziemski, M. M. Marinak, G. D. Kerbel, D. N. Bittner

July 8,2005

Fusion Science and Technology 
This document was prepared as an account of work sponsored by an agency of the United States Government. Neither the United States Government nor the University of California nor any of their employees, makes any warranty, express or implied, or assumes any legal liability or responsibility for the accuracy, completeness, or usefulness of any information, apparatus, product, or process disclosed, or represents that its use would not infringe privately owned rights. Reference herein to any specific commercial product, process, or service by trade name, trademark, manufacturer, or otherwise, does not necessarily constitute or imply its endorsement, recommendation, or favoring by the United States Government or the University of California. The views and opinions of authors expressed herein do not necessarily state or reflect those of the United States Government or the University of California, and shall not be used for advertising or product endorsement purposes. 


\title{
LOW MODE CONTROL OF CRYOGENIC ICF FUEL LAYERS USING INFRARED HEATING
}

\author{
R. A. London, B. J. Kozioziemski, M. M. Marinak, G. D. Kerbel \\ University of California, Lawrence Livermore National Laboratory \\ 7000 East Avenue, Livermore, CA 94550 \\ email: rlondon@1lnl.gov \\ and \\ D. N. Bittner \\ Schafer Corporation \\ 303 Lindbergh Avenue, Livermore, CA 94551
}

\begin{abstract}
Infrared heating has been demonstrated as an effective technique to smooth solid hydrogen layers inside transparent cryogenic inertial confinement fusion capsules. Control of the first two Legendre modes of the fuel thickness perturbations using two infrared beams injected into a hohlraum was predicted by modeling and experimentally demonstrated. In the current work, we use coupled ray tracing and heat transfer simulations to explore a wider range of control of long scale length asymmetries. We demonstrate several scenarios to control the first four Legendre modes in the fuel layer using four beams. With such a system, it appears possible to smooth both short and long scale length fuel thickness variations in transparent indirect drive inertial confinement fusion targets.
\end{abstract}

\section{INTRODUCTION}

The conventional method to produce a smooth layer of solid fuel within an inertial confinement fusion (ICF) capsule is known as beta-layering. ${ }^{1,2}$ In this process, the energy from tritium beta decay causes local heating of the deuterium-tritium (DT) fuel. This leads to sublimation of DT from warmer regions of the inner fuel surface and condensation onto colder regions. In a spherically symmetric temperature environment, this produces a symmetric fuel layer. Infrared (IR) radiation can be used to supplement the beta-decay heating for IR transmissive capsules (such as $\mathrm{CH}$ ) containing tritium (i.e. DT), or to replace the beta-decay heating in ICF capsules without tritium (HD or $\left.\mathrm{D}_{2}\right)^{3,4}$ If the capsule environment is not symmetric, uniform heating of the fuel will generate long spatial scale length (low mode number) thickness perturbations. The indirect-drive ICF design places the spherical fuel capsule inside a cylindrical hohlraum. Elongation of a typical hohlraum (length $\approx 1.7 \mathrm{x}$ width) and the presence of laser entrance holes at both ends leads to an asymmetric temperature field in which the equator of the capsule is cooler than the poles. With uniform heating, as from beta-decay, this leads to a layer that is thicker at the equator than the poles. This asymmetry degrades the convergence of the laser driven implosion and thereby reduces the fusion yield. One method to control asymmetry involves creating a temperature gradient along the hohlraum wall-with the center of the hohlraum being hotter than the ends-by applying heat to the wall. ${ }^{5}$

Another method to control low mode asymmetry uses IR irradiation. Recently, it was demonstrated that one can control the two lowest modes of $\mathrm{D}_{2}$ thickness perturbations using two properly pointed IR laser beams. ${ }^{6,7}$ Each beam was a ring, symmetric about the hohlraum axis. The beams irradiated a small annular surface area on the inside hohlraum wall. The hohlraum walls were roughened in order to create a diffuse scattering surface. The scattered radiation then irradiated the capsule. By using an IR wavelength coincident with an absorption line in $\mathrm{D}_{2}$, the IR was preferentially absorbed in the solid fuel layer. To remove the lowest order perturbation-a $\mathrm{P}_{1}$ Legendre mode-we require that the power and pointing of the two beams relative to the hohlraum center be equal. With two beams - one entering each laser entrance hole - the second order mode, $\mathrm{P}_{2}$, can be removed by proper pointing of the beams relative to the midplane of the hohlraum.

In the present work, we explore the removal of higher order perturbations by using more IR beams. In particular, we explore the use of four beams to simultaneously remove the $\mathrm{P}_{2}$ and $\mathrm{P}_{4}$ perturbation modes. We assume that the odd modes can be controlled by stringent symmetry of the targets and the opposing IR beams. We apply a computational model describing the transport of IR radiation in the hohlraum and thermal conduction of the heat deposited by both beta-decay and IR absorption. The pointing and power of each beam are the controllable parame- 
ters. We use the model to search for values of these parameters that provide a sufficiently symmetric thermal environment to meet the DT fuel layer uniformity required for successful ICF implosion.

\section{COMPUTATIONAL MODEL}

\section{A. Problem Geometry}

We assume that the target and IR irradiation system are symmetric about the midplane and azimuthally sym- metric about the hohlraum axis, as shown in Figure 1. The computational domain is thereby reduced from a full 3-D space to a cylindrical half-space. We model only two IR beams, assuming a matched pair symmetric about the midplane of the hohlraum. Previous simulations indicate that the pointing offset perpendicular to the hohlraum axis must be less than $100 \mu \mathrm{m}$ to maintain azimuthal symmetry of the fuel layer. ${ }^{6}$ The target dimensions, beam parameters and other physical data are given in Table I.

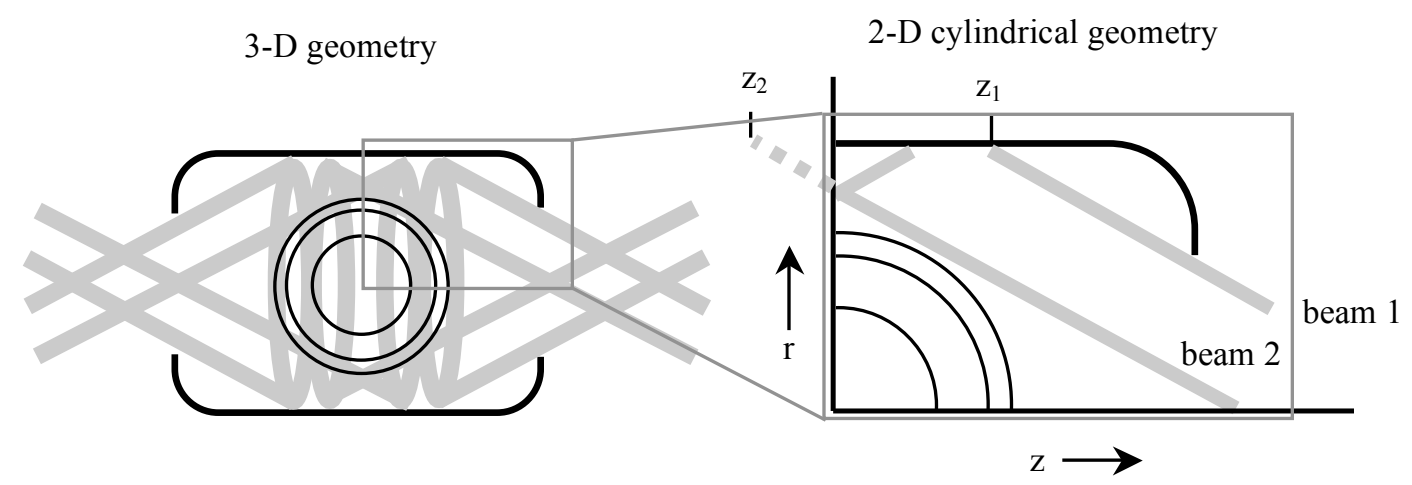

Figure 1. The 3-D geometry with 4 IR beams is reduced to a cylindrical half-space with 2 beams. We show the hohlraum, the capsule, the fuel layer, and the IR beams, with pointing values indicated as $z_{1}$ and $z_{2}$.

Table I. Target Parameters and Physical Data

\begin{tabular}{|c|c|}
\hline hohlraum radius & $2.75 \mathrm{~mm}$ \\
\hline hohlraum length & $9.5 \mathrm{~mm}$ \\
\hline laser entrance hole radius & $1.375 \mathrm{~mm}$ \\
\hline capsule outer radius & $1.0 \mathrm{~mm}$ \\
\hline capsule thickness & $0.15 \mathrm{~mm}$ \\
\hline fuel thickness & $0.10 \mathrm{~mm}$ \\
\hline hohlraum gas conductivity ${ }^{8}$ & $2.0 \times 10^{-5} \mathrm{~W} /(\mathrm{mm}-\mathrm{K})$ \\
\hline capsule $(\mathrm{CH})$ conductivity $^{9}$ & $1.5 \times 10^{-4} \mathrm{~W} /(\mathrm{mm}-\mathrm{K})$ \\
\hline fuel (DT) conductivity ${ }^{10}$ & $2.4 \times 10^{-4} \mathrm{~W} /(\mathrm{mm}-\mathrm{K})$ \\
\hline IR wavelength & $3.46 \mu \mathrm{m}$ \\
\hline IR beam angle (wrt z-axis) & 20 degrees \\
\hline capsule absorption coeff. ${ }^{11}$ & $1.0 \mathrm{~mm}^{-1}$ \\
\hline fuel absorption coeff. ${ }^{12}$ & $0.1 \mathrm{~mm}^{-1}$ \\
\hline capsule refractive index ${ }^{13 . a}$ & 1.59 \\
\hline fuel refractive index ${ }^{14}$ & 1.15 \\
\hline
\end{tabular}

${ }^{a}$ We use an optical wavelength refractive index. The index at $3.46 \mu \mathrm{m}$ is uncertain but expected to be 5$10 \%$ smaller. Calculations show that such a difference does not significantly affect the results.

\section{B. Computer Program}

We simulate the thermal environment of the cryogenic ICF target using the HYDRA computer program. HYDRA is a two and three-dimensional multi-physics program, developed at LLNL to model capsule implosions and hohlraum dynamics for ICF. ${ }^{15}$ We have made several changes to enable cryogenic simulations. The laser ray-tracing package was modified to model the transport of IR radiation. The electron heat transfer package was adapted to model the atomic and molecular heat conduction, with user specified conductivities.

The ray tracing modifications are described in more detail. A large number of rays are traced through the system. Each ray is assigned an energy that decreases due to volumetric absorption and absorption at scattering surfaces. Rays are tracked until their energy is reduced to a specified fraction of their initial energy, typically $1 \%$, or they exit the hohlraum. Wall scattering and interface interactions are treated with a Monte Carlo method. Physical models are used to choose the scattering angle when a ray hits the hohlraum wall and to determine whether a ray is reflected or refracted at an interface where the refractive index changes. The Monte Carlo method produces a statistical realization of the physical models, using weighted random numbers. The angular distribution upon scattering from the roughened hohlraum wall is described 
by the bidirectional scattering distribution function (BSDF). The BSDF gives the radiance of scattered light as a function of the scattering direction, relative to the incident irradiance. It has units of inverse solid angle (steradians). In HYDRA, we use an empirical formula for the BSDF called the Harvey-Shack scattering formula: ${ }^{16}$

$$
B S D F=\frac{A}{B+\left|\vec{\beta}-\vec{\beta}_{0}\right|^{g}},
$$

where $\vec{\beta}$ is the projection on the scattering surface of a unit vector in the scattering direction, while $\vec{\beta}_{0}$ is the projection of a unit vector in the specular direction to the incident ray. The parameters $A, B$, and $g$ are fit to data for specific surfaces. A Lambertian surface (BSDF $=$ constant) can be modeled with $g=0$. The parameters are specified for a number of incident angles, and interpolated for angles between the specified ones. The parameter values used in the present work have been taken from measurements of roughened gold surfaces ${ }^{7}$ and are listed in Table II. In addition to the scattering model, we have implemented user specified IR absorption coefficients for each material, specifically for the fuel and capsule, and Fresnel reflections at material boundaries, according to user supplied index of refraction data.

Table II. Hohlraum wall scattering parameters

\begin{tabular}{|l|l|l|l|}
\hline inc. angle & $A$ & $B$ & $g$ \\
\hline 10 & 0.128 & 0.346 & 1.523 \\
\hline 40 & 0.115 & 0.322 & 1.184 \\
\hline 70 & 0.06 & 0.0765 & 1.168 \\
\hline
\end{tabular}

Previously we performed simulations of IR layering of cryogenic targets with two separate codes-one for ray tracing and one for heat conduction. ${ }^{6}$ Linking the heat deposition from the ray tracing code to the conduction code required a third code and a large effort. Performing simulations with HYDRA has the advantage of allowing all of the physics to be modeled in one code, and it also allows us to run on massively parallel supercomputers.

\section{C. Simulation Methodology}

Variations in the fuel layer thickness around the capsule can result in an anisotropic implosion and low fusion yield. The maximum allowable perturbation amplitude is a function of mode number, and has been determined from a combination of measured amplitudes and numerical implosion simulations. ${ }^{17}$ The current specification allows a maximum thickness perturbation in the $\mathrm{P}_{2}$ and $\mathrm{P}_{4}$ modes (root-sum-square) of $\Delta r_{r s s}=0.57 \mu \mathrm{m}$. The design goal for the IR system is to keep the layer thickness variation less than this value.
To obtain IR system designs that satisfy the fuel layer uniformity goal, we search a 4-D parameter space consisting of the pointing and power values for each of two beams. For each set of parameters we calculate the thickness perturbation as follows. The essential physics of beta and/or IR layering dictates that the DT will redistribute itself until the inner surface is isothermal. Since HYDRA does not simulate the sublimation and recondensation of the fuel, we use the temperature variation assuming a uniform layer thickness as a surrogate for the thickness variation expected after redistribution, when the layer surface becomes isothermal. We show the calculation of the temperature variation on the inner fuel surface in Figure 2.

The relationship between the thickness variation at constant temperature and the temperature variation at constant thickness is determined by calculations for fixed beam parameters in which we manually perturb the DT surface and calculate the departure from isothermality. We perform several calculations of this type using a Newton-Raphson iteration to find the thickness perturbation that produces an isothermal inner DT surface. It is proportional to the temperature at constant thickness:

$$
\Delta r_{r S s}=0.19 \mu \mathrm{m} \frac{\Delta T_{r S S} /\langle T\rangle}{10^{-5}},
$$

where $\langle T\rangle$ is the average temperature of the inner DT surface relative to the hohlraum wall. Combining Eq. (2) with the maximum thickness variation given above $(0.57$ $\mu \mathrm{m})$ we find the maximum allowed temperature variation:

$$
\Delta T_{r S S} /\langle T\rangle \leq 3 \times 10^{-5}
$$

We utilize the linearity of the model to efficiently explore the large parameter space. The temperature caused by each beam is additive and proportional to the beam power. This is true because all of the physical data (conductivity, IR absorption coefficients, etc) are essentially constant in the small temperature range under consideration $( \pm 1 \mathrm{~K})$. We therefore write the inner fuel temperature as a linear combination of three terms:

$$
T=T_{0}+T_{1}+T_{2},
$$

where $T_{0}$ is the temperature due to beta-decay heating, $T_{1}$ the temperature due to the first IR beam and $T_{2}$ the temperature due to the second beam. Due to the assumed azimuthal symmetry, $T$ is a function of only the polar angle from the hohlraum axis, $\theta$.

We express each of the 3 temperature components as a 3 -term Legendre series in $x=\cos \theta$ :

$$
T_{m}=a_{0, m}+a_{2, m} P_{2}(x)+a_{4, m} P_{4}(x),
$$

where $\mathrm{m}=0,1$, or 2 as described below Eq. (4). 

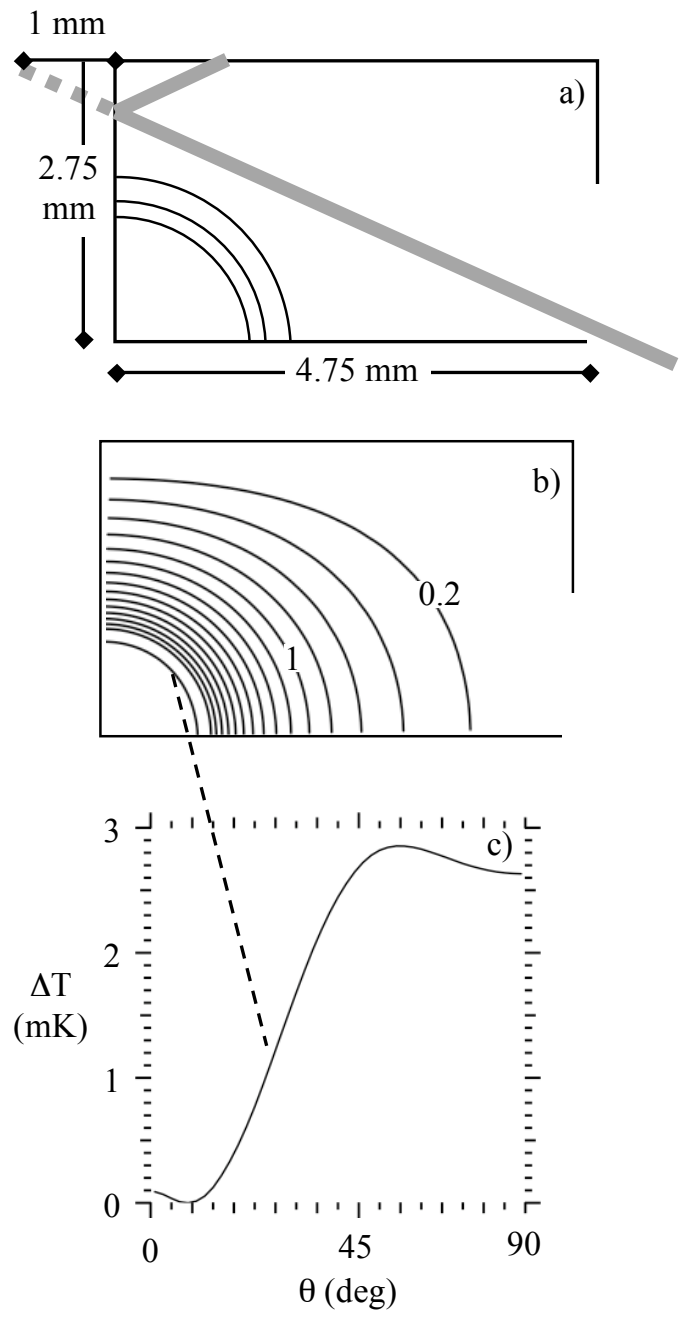

Figure 2. The temperature on the inner surface of the fuel is shown for a particular pointing of a single beam. The calculation is for an IR power of 300 times the beta-decay power, or $12 \mathrm{~mW}$. Panel a) shows the hohlraum dimensions and the beam pointing. Panel $b$ ) shows temperature contours in the hohlraum gas, the capsule, and the fuel. The contour interval is $0.2 \mathrm{~K}$. The maximum temperature is $3.1 \mathrm{~K}$ above the hohlraum wall temperature. Panel c) shows the temperature variation on the inner fuel surface, with $\theta=0$ corresponding to the hohlraum axis (the "pole").

To calculate the Legendre coefficients, we perform simulations of the IR transfer and heat conduction with HYDRA for single beam irradiation at different pointings. We measure the pointing relative to the mid-plane of the target with negative numbers corresponding to pointing beyond the midplane from the laser entrance hole. We have performed 9 simulations with pointing values between -2 and $1 \mathrm{~mm}$. The beam is incident at an angle of $20^{\circ}$ with respect to the hohlraum axis. Each beam is chosen to be very thin $(0.01 \mu \mathrm{m}$ wide). Thin beams are used to provide the maximum flexibility in combining beams to produce arbitrary irradiation patterns. After acceptable solutions are found, we repeat calculations with specific pointings with more realistic (wider) beams as described in §III B. For each simulation, we calculate the normalized Legendre coefficients of the inner DT surface temperature:

$$
\alpha_{2}(z)=\frac{a_{2}(z)}{a_{0}(z)}, \alpha_{4}(z)=\frac{a_{4}(z)}{a_{0}(z)},
$$

where $\mathrm{z}$ indicates the pointing value. In addition to the IR calculations, we performed a HYDRA calculation for pure beta-decay heating to determine its contribution to the asymmetry.

Figure 3 shows the variation of the normalized mode coefficients, $\alpha_{2}$ and $\alpha_{4}$, with beam pointing and the mode coefficients for beta heating. The $\mathrm{P}_{2}$ coefficient decreases for pointing between -2 and $0 \mathrm{~mm}$ and then becomes relatively flat for pointing values between 0 and $1 \mathrm{~mm}$. The $\mathrm{P}_{4}$ coefficient is flat between -2 and -1 , increases between -1 and 0.3 and is then flat again between 0.3 and 1 $\mathrm{mm}$. The $\mathrm{P}_{2}$ due to beta heating is positive and relatively large, while the $\mathrm{P}_{4}$ is quite small.

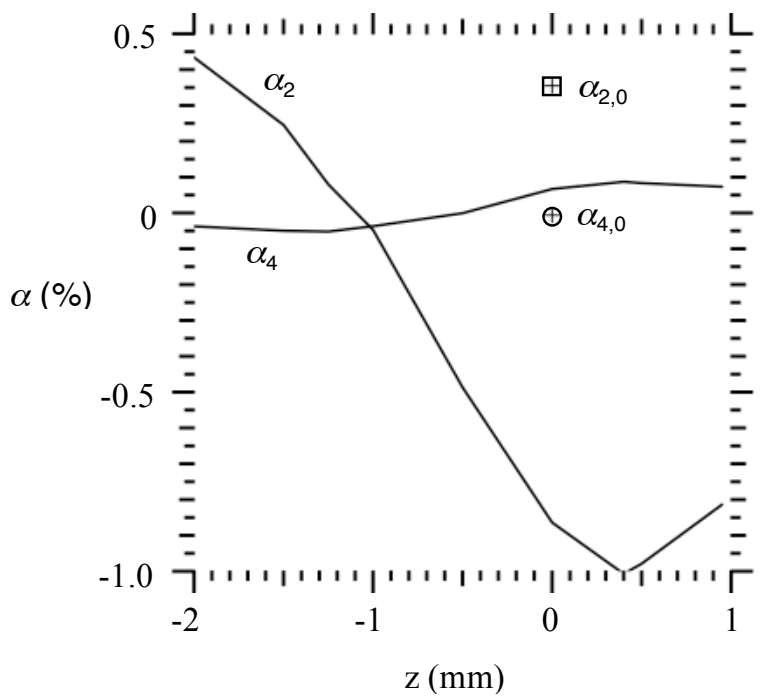

Figure 3. The normalized values of the $\mathrm{P}_{2}$ and $\mathrm{P}_{4}$ coefficients of the temperature on the inner fuel surface $\left(\alpha_{2}, \alpha_{4}\right)$ are shown versus the IR beam pointing, z. The coefficients for beta-decay heating $\left(\alpha_{2,0}, \alpha_{4,0}\right)$ are the crossed circle and square, plotted arbitrarily at $\mathrm{z}=0$.

To reduce the parameter space search from 4-D to 3$\mathrm{D}$, we first minimize the $\mathrm{P}_{2}$ perturbation. For each pair of pointing values and total IR power, we solve for the beam powers to zero the total $\mathrm{P}_{2}$ coefficient. This involves solving the following two equations: 


$$
\alpha_{2,0} a_{0,0}+\alpha_{2,1} a_{0,1}+\alpha_{2,2} a_{0,2}=0
$$

and

$$
W_{1}+W_{2}=W_{i r} .
$$

Here $W_{1}$ is the power of beam 1 (similar for beam 2) and $W_{\text {ir }}$ is the total (fixed) IR power. We must supplement these equations by the linear scaling factors between the IR power $\left(W_{m}\right)$ and the zero-order mode amplitude $\left(a_{0, m}\right)$ for each beam. These factors are found from the particular input and output of each HYDRA simulation. If the solution of Eqs. (7-8) produces a negative power for either beam, we zero that power and set the other beam power equal to the total IR power.

\section{RESULTS}

\section{A. Parameter Survey}

We now search the 3-D parameter space by stepping through a small number of IR power values and performing a high-resolution scan of the 2 beam pointing values for each IR power. We have examined 12 power values and 200 pointing values for each beam. To avoid redundancy, we restrict $z_{2}$ to be greater than $z_{1}$. The IR power is expressed in terms of the beta-decay power, which, in DT, is $40 \mu \mathrm{W}$ for the fuel layer dimensions given in Table I. From the HYDRA simulations, we find that approximately $1 \%$ of the IR power is absorbed in the fuel, $10 \%$ in the plastic shell, and $52 \%$ in the hohlraum walls. About $37 \%$ of the IR power escapes the hohlraum.

Figures 4a-d show contours of the temperature variation versus the beam pointings for four values of the total IR power. We have shown only the region in which interesting solutions are found. Each position in the $z_{l}-z_{2}$ planes shown in Figure 4 is associated with beam powers that add up to the fixed power for that panel. The beam power ratios are discussed below. At the very lowest IR power $\left(W_{i r}=5 W_{\beta}\right)$, Figure 4 a shows that there is only a small region for which the temperature variation is small enough. At slightly higher IR power (4b) the acceptable region opens up with either beam pointed near $-0.4 \mathrm{~mm}$. With increasing IR power, the acceptable region broadens and moves toward smaller values of $z_{l}$ and larger values of $z_{2}$. The largest acceptable region occurs for $W_{i r} \approx 10 W_{\beta}$ (4c). As $W_{i r}$ is further increased the acceptable region shrinks and moves to the left, toward a pointing of $z_{l}=-2$ $\mathrm{mm}$ (Figure 4d).

\section{B. Low and High Power Solutions}

We now take a closer look at the solutions for two power levels. In Figure 5 we show the contours of the beam power ratio, as determined by Eqs. (7-8) to minimize the $\mathrm{P}_{2}$ variation as well as the region of acceptable temperature variation. Figure 5 a shows the case for $W_{i r}=$ $10 W_{\beta}$. The IR power absorbed in the fuel is only $10 \%$ of the beta decay power, not sufficient to affect the layering rate or the higher mode roughness. However, there is still sufficient IR heating in the plastic shell $\left(\approx W_{\beta}\right)$ to allow control of the low mode symmetry. In fact, we find a wide range of pointing values that give acceptable solutions. One of the solutions for $W_{i r}=10 W_{\beta}$ is indicated by a white dot on Figure 5a and detailed in Table III.

At high power $\left(W_{i r}=300 W_{\beta}\right.$, Figure $\left.5 \mathrm{~b}\right)$ the IR heating in the fuel is significant $\left(3 W_{\beta}\right)$. This power level is to be considered if the beneficial effects of increased layering rate and enhanced higher mode smoothing are desired. ${ }^{3,4,7}$ This power is close to the maximum that can be removed through the hohlraum gas and cryogenic cooling rods. From Figure 5b, we find solutions for high power with beam 1 pointed near the limiting value of $-2 \mathrm{~mm}$ and beam 2 in the range $0-1 \mathrm{~mm}$. One solution is marked by a white dot on Figure $5 b$ and detailed in Table III.

Table III. Low and High Power Solutions

\begin{tabular}{|c|c|c|}
\hline$W_{i r} / W_{\beta}$ & 10 & 300 \\
\hline$W_{2} / W_{1}$ & 0.528 & 0.331 \\
\hline$z_{1}(\mathrm{~mm})$ & -1.25 & -1.97 \\
\hline$z_{2}(\mathrm{~mm})$ & 0.38 & 0.37 \\
\hline
\end{tabular}

All of the solutions illustrated in Figures 4 and 5 assumed thin IR beams. In reality, one would use wider beams. ${ }^{7}$ We have done of calculations for $100 \mu \mathrm{m}$ wide (FWHM) beams for the cases summarized in Table III. For both cases we find acceptable solutions (with small temperature variations), with power ratios only slightly different from those specified for the pencil beams. The use of realistic wide beams does not greatly change the optimal designs.

\section{C. Required Accuracy}

We now discuss the necessary beam pointing accuracy. Although a first glance at Figures 4 and 5 suggests that a rather large range of acceptable beam pointings, it should be emphasized that the power ratio has been changed as the beam pointings are changed in these Figures. For an experiment, it is more realistic to consider the situation with a fixed power ratio. Figure 6 shows temperature variation contours for the low power solution, $\left(W_{i r}=10 W_{\beta}\right)$ with the power ratio fixed at the value given in Table III. Now we see a much large sensitivity to beam pointing. At the optimal position for this power ratio, in- 
dicated by the white circle, the maximum allowed change in pointing is about $\pm 27 \mu \mathrm{m}$ for beam 1 and $\pm 123 \mu \mathrm{m}$ for beam 2. Although Figure 6 appears to show that the acceptable range of $z_{2}$ is much smaller for other values of $z_{1}$, this is misleading. A slight adjustment of the power ratio can be made to re-optimize the solution and give ap- proximately the same allowable range of beam pointings as for the optimal case. The high power solution listed in Table III has similar constraints on the pointing accuracy. A similar analysis shows that the power of each beam must be accurate to $\pm 2.3 \%$ to obtain an acceptably small temperature perturbation.
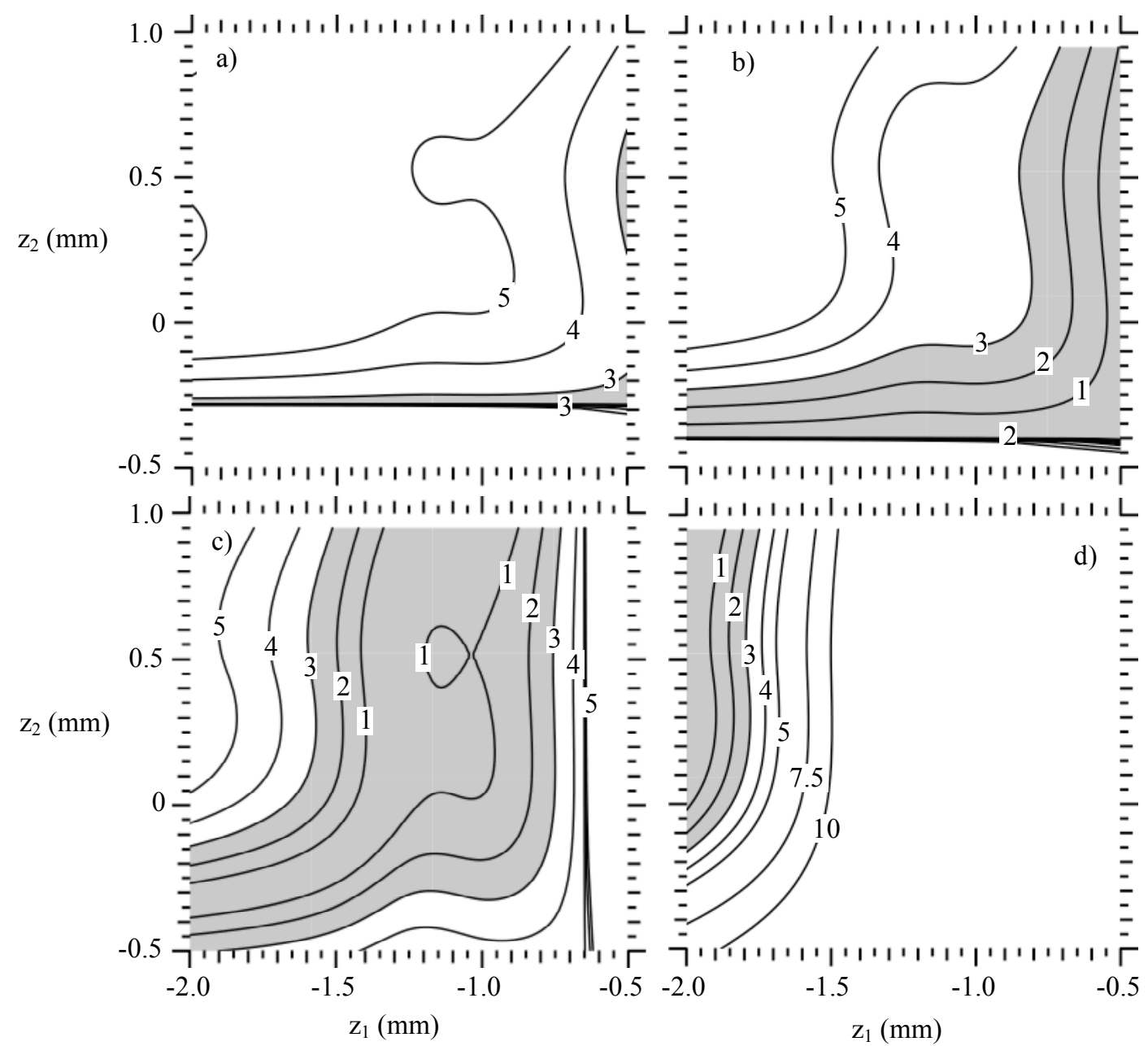

Figure 4. Contours of the normalized temperature variation, $\Delta T /<T>$, are shown versus the IR beam pointing, $z_{1}$ and $z_{2}$. The contours are labeled in units of $10^{-5}$. The acceptable region $\left(\Delta T /<T><3 \times 10^{-5}\right)$ is shaded gray on each panel. The total IR powers relative to the beta-decay power, $W_{i r} / W_{\beta}$ are $5,6,10$, and 300 , for panels a)-d), respectively.

\section{D. Single Beam Solution}

Although, we have focused attention on solutions with two IR beams per side, it is instructive to see how well the $\mathrm{P}_{2}$ and $\mathrm{P}_{4}$ modes can be controlled with a single IR beam per side. To do so we have calculated the total temperature perturbation, $\Delta T_{r s s}$, as a function of the beam power and pointing. We find acceptable solutions in a small region of parameter space at low IR power, with $W_{i r}=4.9$ to $8 W_{\beta}$, and $z=-0.55$ to $-0.27 \mathrm{~mm}$. At the opti- mal position $\left(W_{i r}=6 W_{\beta}, z=-.41 \mathrm{~mm}\right)$, the allowable pointing range is $\pm 13 \mu \mathrm{m}$ and the allowable power range is $\pm 2.2 \%$. This solution is somewhat less robust (i.e. better accuracy is required) than the low power solutions with two beams discussed in §III B. In contrast to the two-beam case, there are no single beam solutions for larger IR power. Therefore, the use of two beams allows more solutions and eases the pointing and power accuracy requirements. 


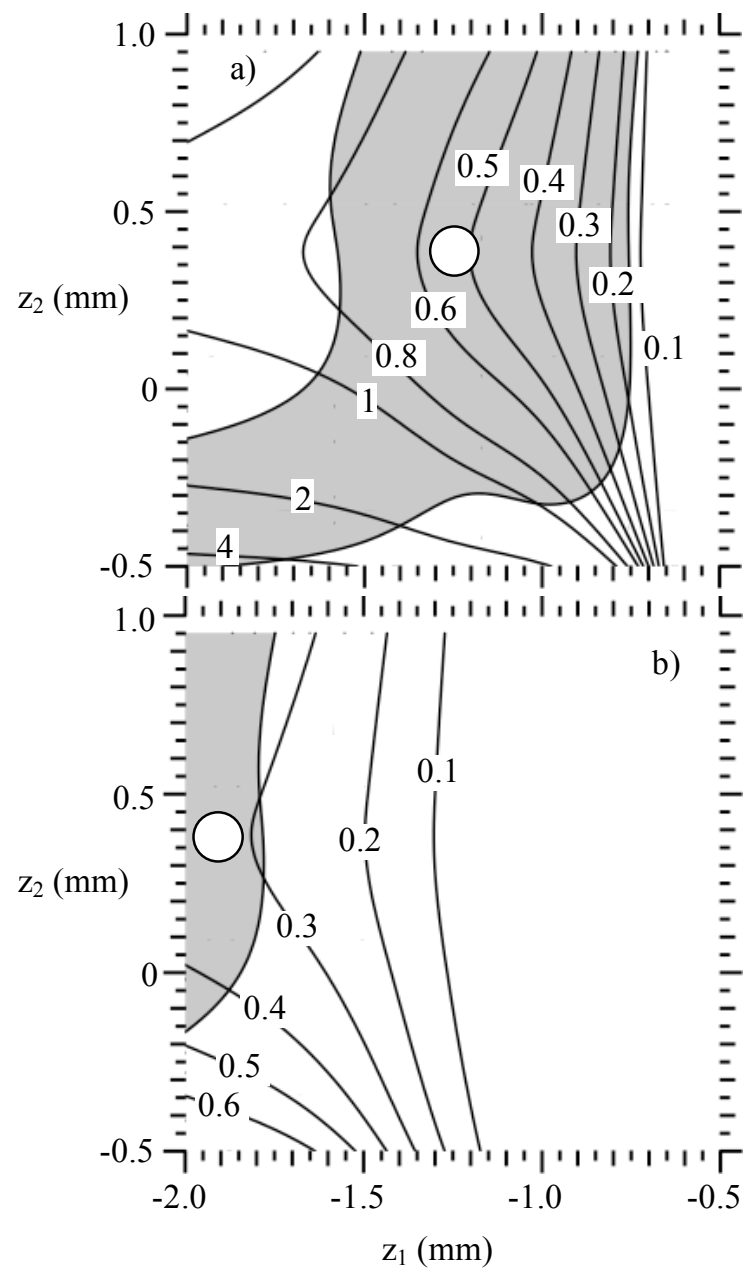

Figure 5. Contours of the beam power ratio, $W_{2} / W_{1}$, are shown versus beam pointing, $z_{1}$ and $z_{2}$. The total powers are $W_{i r} / W_{\beta}=10$ and 300 for panels a) and b), respectively. The shaded area on each plot is the region of acceptable temperature variation $\left(\Delta T /<T><3 \times 10^{-5}\right)$. Circles mark typical solutions, detailed in Table III.

\section{IMPLEMENTATION}

Experiments to date have been done with two IR beams using a pair of custom designed axicons to inject a single ring of light through each hohlraum laser entrance hole $^{7}$. The output of an IR laser was fiber coupled to an axicon and shaped by the axicon into a focused ring for injection into the hohlraum. For injecting two (or more) rings of light through each laser entrance hole, a series of axicons is impractical. Shaping a beam from each side into the equivalent of multiple single beam injections would be required. Since the injection optics would need to be mounted on a cryostat, minimizing the size of the optical system is important.

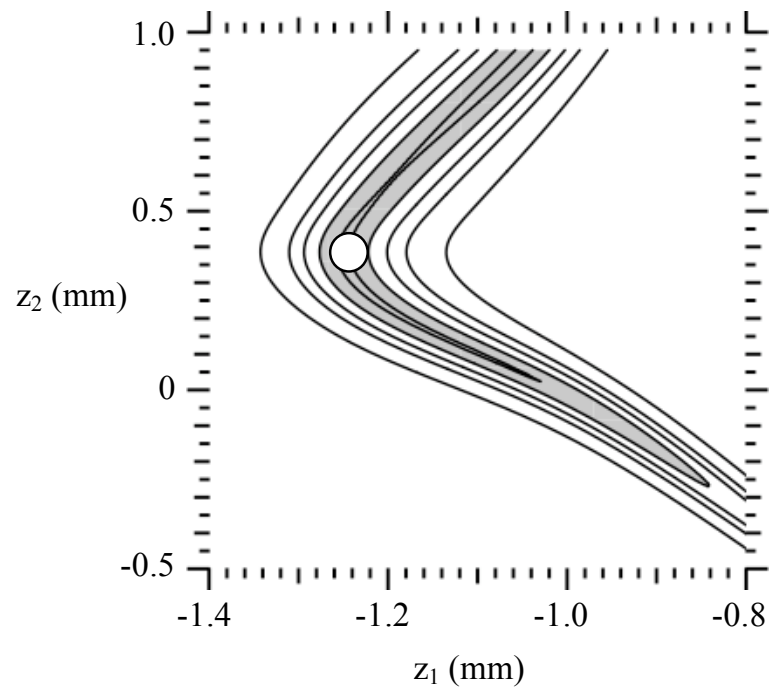

Figure 6. Contours of the normalized temperature variation, $\Delta T /<T>$, are shown versus the pointing of the two IR beams, $z_{l}$ and $z_{2}$ with fixed power ratio $=0.528$. The contour levels range from $10^{-5}$ to $11 \times 10^{-5}$, in equal steps. The total IR power is $10 W_{\beta}$. The shaded area is the region of acceptable temperature variation $\left(\Delta T /<T><3 \times 10^{-5}\right)$. The white circle marks the optimal position. The acceptable variations in beam positions about the optimal are $\Delta z_{l}=$ $\pm 27 \mu \mathrm{m}$ and $\Delta z_{2}= \pm 123 \mu \mathrm{m}$.

Diffractive optics technologies appear to provide the ability to form light into a variety of shapes in a compact optical package as indicated by their wide spread use in numerous applications including beam splitters, beam shapers, and beam diffusers.

To implement the 4-beam injection system discussed in this paper, we propose the use of two diffractive optical elements-one to provide 2 beams for each laser entrance hole. Each diffractive element would take the output from an optical fiber and generate the 2 beams discussed above. This technique could be extended to more than 2 beams or a beam with a continuously varying intensity. According to the design calculations presented in §III, the system would be required to deliver a maximum of approximately $12 \mathrm{~mW}$ of IR power into the hohlraum. The pointing accuracy and stability would have to be about $\pm 27 \mu \mathrm{m}$ along the hohlraum wall, and the power ratio accuracy would have to be about $\pm 2.3 \%$. The pointing and power values determined by calculations such as those presented here would be used to guide an experiment to determine the actual values appropriate to achieve an acceptably uniform fuel layer. 


\section{CONCLUSIONS}

We have presented a computational study of the control of low mode fuel layer symmetry in indirect drive ICF capsules using a four beam IR irradiation system. The assumption of azimuthal and reflection symmetry enables the consideration of just two independent beams and even perturbation modes. We have found a multitude of solutions for the 4 parameters describing the system (the powers and pointing positions of each of the 2 beams). In particular, solutions are found at low power, where the main effect of the IR is to control the symmetry by heating the capsule, and at high IR power, where the IR both controls the symmetry and heats the fuel layer thereby enhancing the layering process. We propose an implementation of such an IR irradiation system using diffractive optical elements.

\section{ACKNOWLEDGMENTS}

We are pleased to thank J. Moody, J. Sanchez, and J. Sater for helpful discussions. This work was performed under the auspices of the U.S. Department of Energy by the University of California, Lawrence Livermore National Laboratory under contract W-7405-Eng-48.

\section{REFERENCES}

1. A. J. MARTIN, R. J. SIMMS and R. B. JACOBS, "Beta Energy Driven Uniform Deuterium-Tritium Ice Layer in Reactor-Size Cryogenic Inertial Fusion Targets,” J. Vac. Sci. Technol., A6, 1885 (1988).

2. J. K. HOFFER and L.R. FOREMAN, "Radioactively Induced Sublimation in Solid Tritium," Phys. Rev. Lett., 60, 1310 (1988).

3. G. W. COLLINS, D. N. BITTNER, E. MONSLER, S. LETTS, E. R. MAPOLES, and T. P. BERNAT, "Infrared Redistribution of D2 and HD Layers for Inertial Confinement Fusion," J. Vac. Sci. Technol., A14, 2897 (1996).

4. B. J. KOZIOZIEMSKI, R. L. MCEACHERN, R. A. LONDON, and D. N. BITTNER, "Infrared Heating of Hydrogen Layers in Hohlraums," Fusion Sci. Technol., 41, 296 (2002).

5. J. J. SANCHEZ and W. H. GIEDT, "Thermal Control of Cryogenic Cylindrical Hohlraums for Indirect-Drive Inertial Confinement Fusion," Fusion Technol., 36, 346 (1999).
6. R. A. LONDON, R. L. MCEACHERN, B. J. KOZIOZIEMSKI, and D. N. BITTNER, "Computational Design of Infrared Enhanced Layering of ICF Capsules," Fusion Sci. Technol. 45, 245 (2004).

7. B. J. KOZIOZIEMSKI, R. A. LONDON, R. L. MCEACHERN, and D. N. BITTNER, "Demonstration of Symmetry Control of Infrared Heated Deuterium Layers in Hohlraums," Fusion Sci. Technol., 45, 262 (2004).

8. J. G. WEISAND II, ed., Handbook of Cryogenic Engineering, p. 25 (Taylor and Francis, Philadelphia, 1998).

9. G. HARTWIG, Polymer Properties at Room and Cryogenic Temperatures, p 63 (Plenum Press, New York, 1994).

10. G. W. COLLINS, P. C. SOUERS, E. M. FEARON, E. R. MAPOLES, R. T. TSUGAWA, and J. R. GAINES, "Thermal conductivity of condensed D-T and $\mathrm{T}_{2}, "$ Phys. Rev. B, 41, 1816 (1990).

11. R. COOK, M. ANTHAMATTEN, S. LetTS, A. NIKROO, and D. CZECHOWICZ, "Infrared Absorptive Properties of Plastic Materials Used in ICF Capsules," Fusion Sci. Technol., 45, 148 (2004).

12. G. W. COLLINS, Lawrence Livermore National Laboratory (private communication).

13. J. C. SEFERIS, "Refractive Indices of Polymers," in Polymer Handbook, p. VI-571 (Wiley, New York, 1999).

14. P. C. SOUERS, Hydrogen Properties for Fusion Energy, p 70 (UC Press, Berkeley, 1986).

15. M. M. MARINAK, G. D. KERBEL, N. A. GENTILE, O. JONES, D. MUNRO, S. POLLAINE, T. R. DITTRICH, and S. W. HAAN, "ThreeDimensional HYDRA Simulations of National Ignition Facility Targets," Phys. Plasmas, 8, 2275 (2001).

16. J. E. HARVEY, "Light Scattering Properties of Optical Surfaces," Ph. D. Dissertation, U. Arizona (1976).

17. S. W. HAAN, et al., "Update on Specifications for NIF Ignition Targets and their Rollup into an Error Budget," Fusion Sci. Technol., (this volume). 